\title{
Evolution récente des terroirs viticoles dans la région do Entre Douro e Minho (nord du Portugal).
}

\author{
Hervé Quénol**** Ana Monteiro*** — Gérard Beltrando** \\ * Laboratoire COSTEL, UMR 6554 du CNRS LETG/Université Rennes 2, Place du \\ recteur Henri Le Moal, 35043 Rennes Cedex (France). \\ herve.quenol@uhb.fr \\ **Equipe Dynamique des Milieux et Risques, UMR 8586 du CNRS \\ PRODIG/Université Denis Diderot Paris 7, 2 place Jussieu 75251 PARIS Cedex 05 \\ (France). \\ beltrando@paris7.jussieu.fr \\ ** Facultade de Letras da Universidade do Porto Departemento da Geografia - \\ Via Panorâmica s $n^{\circ}$, 4150-564, PORTO (Portugal). \\ anamt@letras.up.pt
}

\begin{abstract}
RÉSUMÉ. Depuis plusieurs années, la région do Entre Douro e Minho (EDM) subit une diminution importante de la surface agricole et du nombre d'exploitations. L'analyse des données des Recensements Généraux d'Agriculture (RGA) de 1989 et de 1999 confirme cette tendance mais certaines fréguesias (ensemble de communes) connaissent une augmentation de la surface cultivée pour des cultures comme le Vinho Verde. La représentation spatiale des données des RGA dans un SIG fait ressortir les grandes zones productives de Vinho Verde où les modes de cultures et de production ont évolué vers une démarche d'exportation massive du vin (augmentation de la production et amélioration de la qualité du vin). Cette évolution récente des terroirs viticole a des conséquences sur l'agriculture de la région (diminution de l'agriculture vivrière au profit des grands producteurs de vin) et sur la transformation du paysage.
\end{abstract}

MOTS-CLÉS : vignoble de Vinho Verde, données RGA, SIG, MNT.

ABSTRACT. For several year, "Entre Douro e Minho"(EDM) area has undergone an important diminution in agricultural surface and number of exploitations. RGA analysis of 1989 and 1999 confirms this tendency but some "fréguesias" know an increase in the cultures surface cultivated like Vinho Verde. Spatial representation of the RGA data in a GIS shows the great productive areas of Vinho Verde where cultures and production methods evolved to a wine export politic (production increase and better quality of the wine). This recent evolution of the Vinho Verde changes the EDM agriculture (reduction of "agriculture familiy") and transforms the landscape.

KEYWORDS: Vinho Verde vineyard, RGA data, GIS, DEM.

Nom de la revue. Volume $\mathrm{X}-\mathrm{n}^{\circ} \mathrm{X} / 2005$, pages 1 à $\mathrm{X}$ 


\section{Introduction}

La région do Entre Douro e Minho (DEM) n'est pas une entité administrative mais elle comprend un ensemble de districts représentatifs d'une pratique culturale spécifique. Déterminée par la Direction Générale de l'Agriculture, la région DEM (superficie $8657 \mathrm{~km}^{2}$ ) correspond à la région d'appellation des Vinhos Verdes (Appellation d'Origine Contrôlée créé en 1908) et s'apparente à des paysages composés de champs céréaliers et de "ray-grass" (vignes de Vinho Verde bordant les champs). Le Vinho Verde est produit exclusivement dans le nord du Portugal (également dans le sud de la Galice), il est caractérisé par des variétés de cépages et des conditions biogéogratiques spécifiques. La région des Vinos Verdes est bordée, au nord, par le fleuve Minho ; à l'est et au sud par les montagnes séparant les zones intérieures et à l'ouest, par l'Atlantique (Galhano, 1986). Située au nord ouest du Portugal, elle se définit par un vaste amphithéâtre tourné vers l'océan. Des montagnes de l'intérieur vers le littoral, l'altitude diminue de manière compartimentée. Cet espace est caractérisé par un relief accidenté, dont les altitudes croissent au fur et à mesure que l'on s'éloigne de l'océan Atlantique (a Serra da Peneda-Soajo 1416 m). Les fleuves (ex : Minho et Lima), d'orientation Est-ouest se caractérisent par de larges vallées se resserrant progressivement vers l'intérieur du pays (cf. fig 1).

Depuis quelques années, cette région do Entre Douro e Minho, caractérisée par un espace principalement rural, subit d'importantes transformations. La forte diminution de l'agriculture, tant en nombre d'agriculteurs (-25\% entre 1989 et 1999) qu'en surface cultivée $\left(-30,6 \%\right.$ entre 1989 et $\left.1999^{1}\right)$ ainsi que l'évolution des pratiques culturales (baisse de l'agriculture vivrière et développement de l'agriculture commerciale) engendrent des modifications du territoire et du paysage. Les exploitations de cultures vivrières composées principalement de petites parcelles $<0,5$ ha délimitées par des haies de vignes sont remplacées progressivement par de la forêt, par des parcelles plus grandes destinées aux cultures d'exportation (ex : fruits exotiques, vigne) ou par d'autres activités (ex : industries, ...).

La viticulture qui représente plus de $85 \%$ des cultures permanentes ${ }^{2}$ dans cette région ne déroge pas à cette tendance : diminution du nombre d'exploitations et de la surface cultivée. Les données des derniers RGA (1989-1999) par fréguesia ${ }^{3}$ montrent que la baisse du nombre d'agriculteurs est générale mais que quelques communes connaissent une augmentation non négligeable de la surface en vigne, notamment en cépages permettant la production de Vinho Verde. Ces constatations mettent en évidence un changement des pratiques culturales et une ouverture vers une viticulture plus productive, orientée vers l'exportation, engendrant ainsi le

1. Recenseamentos Gerais da Agricultura, Dados comparativos 1989-1999 Instituto Nacional de Estatistica do Portugal

2. Vigne, oliviers, arbres fruitiers

3 . Équivalent d'un groupement de communes. 
développement de terroirs de plusieurs hectares au détriment de la culture de la vigne sous la forme de haies.

Les politiques (européenne et nationale) et les aides au développement de la vigne du Vinho Verde dans cette région, ont modifié les pratiques culturales et provoqué le développement de grandes exploitations modernes (apparition de plusieurs exploitations supérieures à 50 ha) destinées à produire et à exporter le Vinho Verde. Cette évolution relativement rapide des pratiques agricoles dans le Nord du Portugal n'est pas sans conséquences sur l'économie agricole et sur les paysages ruraux. Les agriculteurs qui produisaient du Vinho Verde de manière «artisanale» ne vinifient plus eux-mêmes (qualité trop moyenne par rapport aux grands producteurs actuels) et ils ont même beaucoup de difficultés à vendre le raisin à la coopérative (quantité trop faible).

Dans cette étude de cas, nous montrons que l'analyse spatiale des données RGA et l'apport des outils de la géomatique - en particulier le Système d'Information Géographique associé à un Modèle Numérique de Terrain - permettent de mettre en évidence la dynamique spatiale récente des terroirs viticoles dans le Nord-Ouest du Portugal.

\section{Bases de données et méthode}

Pour analyser l'évolution de l'agriculture et plus particulièrement de la culture du Vinho Verde dans la région do Entre Douro e Minho, nous avons croisé dans un S.I.G. vectoriel (logiciel Mapinfo), les données issues des Recensements Généraux de l'Agriculture (RGA) de 1989 et 1999, les limites administratives des 1350 fréguesias ainsi qu'un Modèle Numérique de Terrain.

Les fichiers RGA de 1989 et 1999, obtenus auprès de l'Institut National de Statistiques du Portugal, comprennent des informations sur la nature des cultures, la Surface Agricole Utile (SAU), sur le nombre d'exploitants et sur la surface cultivée en vigne de Vinho Verde pour chaque fréguesia. Ces informations ont été associées à une base de données cartographique comprenant les limites administratives des fréguesias de la région administrative do Entre Douro e Minho. Ce fichier a été géoréférencé de manière à pouvoir y combiner un Modèle Numérique de Terrain. La morphométrie est réalisée à partir d'un fichier numérique de courbes de niveau (sources: Instituto Geográfico Portugues) dont l'équidistance est de 25 mètres. Chaque courbe de niveau a été digitalisée et interpolée dans le logiciel Surfer suivant un intervalle de $1 \mathrm{Km}$ de manière à obtenir un champ régulier de points $\mathrm{X}, \mathrm{Y}$ et $\mathrm{Z}$. Ce fichier de points $(X, Y, Z)$ géoréférencé a ensuite été exporté dans le S.I.G. vectoriel sur une grille composée de cellules de $1 \mathrm{Km}^{2}$. Chaque point $\mathrm{X}, \mathrm{Y}$ et $\mathrm{Z}$ est attribué au centroïde de chaque cellule (Bridier et al, 2004). Dans cet article, nous utilisons seulement la visualisation de la topographie en la superposant avec les limites des fréguesias (fig 1). En y associant l'analyse d'images satellites, ce MNT, permettra ultérieurement, d'étudier plus finement l'évolution spatiale des vignobles 
et les impacts paysagers qui en découlent. La grille étant composée d'objets vectoriels, nous pourrons effectuer des requêtes combinant la morphométrie (pente, orientation) avec les limites du vignoble à différentes périodes (analyse multi date d'images satellites).

Les cartes de l'évolution de l'utilisation du sol ont été agrémentées avec des indices de statistique spatiale (indice de ségrégation de Duncan, quotient de localisation et indice I de Moran) afin de valider la signification statistique des structures spatiales observées (Apparicio, 2000).

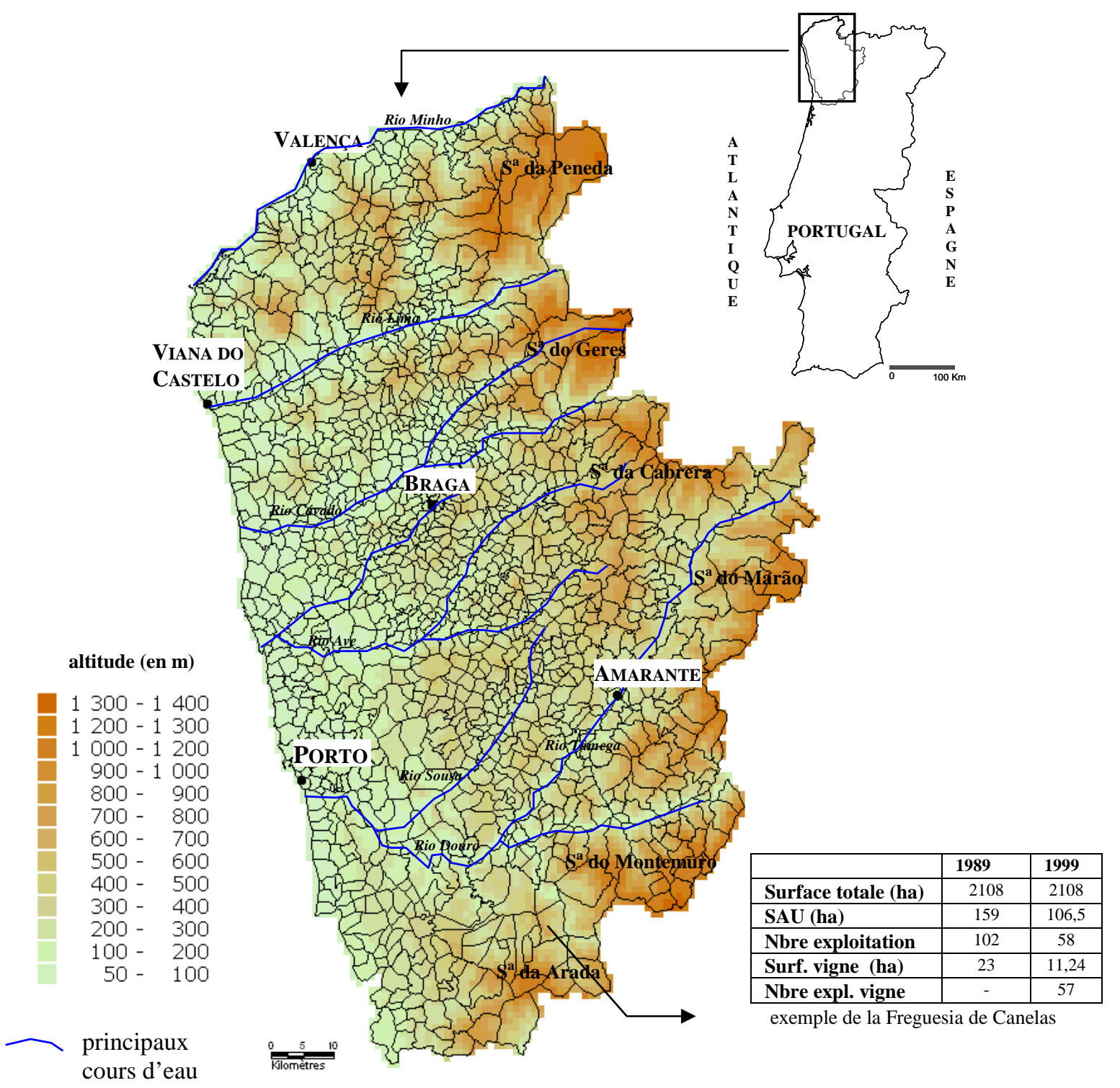

Figure 1. Combinaison des données RGA avec la morphométrie pour chaque fréguesia de la région "do Entre Douro e Minho". 


\section{Les indicateurs de la modernisation de la viticulture dans le Vinho Verde}

\subsection{Une diminution de la surface cultivée : une tendance générale au Portugal et dans la région DEM}

Au Portugal, selon les données de l'Institut National de Statistique (INE) et le Recensement Général de l'Agriculture (RGA) de 1999, 415969 exploitations ont été recensées avec une surface totale de 5188938 ha, représentant approximativement $56 \%$ de la surface du pays. La SAU est de 3863116 ha, soit 75\% de la surface totale des exploitations (tab.1). Entre 1989 et 1999, le nombre d'exploitations agricoles a diminué d'environ $30.6 \%$ et la SAU a baissé de $3.6 \%$. Cette diminution est significative pour les exploitations de moins de 5 ha mais on observe une augmentation des exploitations de plus de $50 \mathrm{ha}^{4}$.

La région do Entre Douro e Minho (DEM) suit la même tendance avec une baisse significative de la surface agricole et du nombre d'exploitations. En 1999, la région EDM constitue une superficie totale de 374832 ha dont 215675 ha de SAU. Entre 1989 et 1999, la SAU a baissé de $10 \%$. Le nombre d'exploitations qui était de 111505 en 1989 atteint 67546 en 1999, soit une diminution de 39,4\%. La région EDM enregistre la plus forte baisse du Portugal.

En 1999, la surface totale agricole, hors SAU, représente 133236 hectares de bois et forêts et 25921 ha pour la surface agricole non utilisée. Après la SAU, qui concerne $57.5 \%$ de la surface totale agricole, la forêt est en seconde position avec $35.5 \%(\operatorname{tab} 1)$.

\begin{tabular}{|c|c|c|c|c|c|}
\hline & $\begin{array}{c}\text { Superficie } \\
\text { totale }\end{array}$ & SAU & Forêts & $\begin{array}{c}\text { Sup. agricole non } \\
\text { utilisée }\end{array}$ & $\begin{array}{c}\text { Nombre } \\
\text { exploitations }\end{array}$ \\
\hline Portugal 99 & 5188938 & 3863094 & 1008374 & 317471 & 415969 \\
\hline EDM 99 & 374832 & 215675 & 133236 & 25921 & 67546 \\
\hline EDM 89 & 464126 & 289710 & 162444 & 11972 & 111505 \\
\hline
\end{tabular}

Tableau 1. Surface totale des exploitations (ha). (Sources : RGA 1989 et 1999).

Les cultures temporaires occupent une grande part de la SAU (près de 50\%) (fig 2). Elles se caractérisent par des prairies et des cultures fourragères. Elles sont associées à la production bovine, qui malgré une forte diminution, représente dans la région EDM encore $22.7 \%$ de la production du pays et de $32.2 \%$ de la production de lait. Les cultures permanentes, (dont la vigne) ont enregistré une forte diminution entre les RGA de 1989 (45 159 ha) et 1999 (33 529 ha) (fig. 3). En 1999, les oliviers et la vigne représentent, au Portugal, respectivement $47 \%$ et $30 \%$ des cultures permanentes. Dans la région EDM, la vigne constitue $85 \%$ du secteur total attribué aux cultures permanentes. Entre les deux recensements (1989 et 1999), la tendance est à une diminution généralisée pour toutes les cultures, à l'exception des fruits

4. Sources : Ministério da Agricultura, do Desenvolvimento Rural e das Pescas (1999). 
tropicaux où il y a une légère augmentation. Celle-ci s'explique par l'essor de la culture du Kiwi destinée à l'exportation (fig 4).

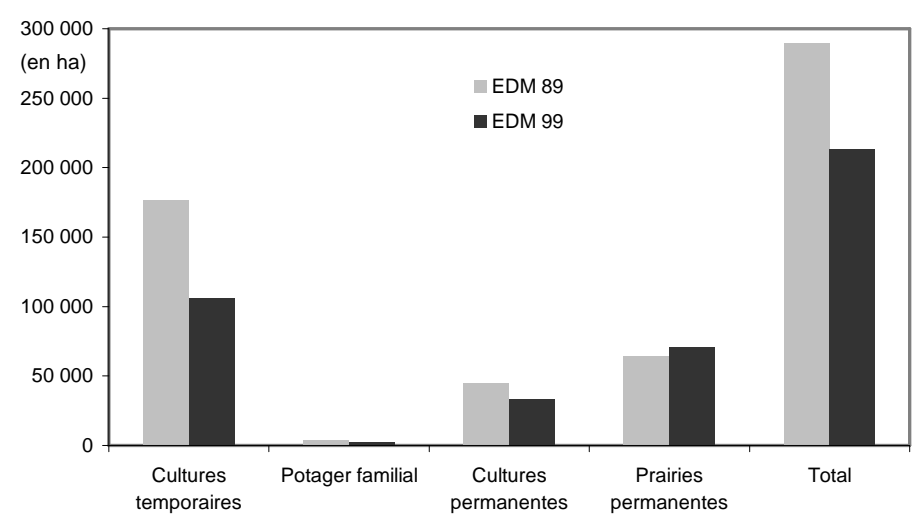

Figure 2. Composition de la SAU (ha). (Sources : RGA 1989 et 1999).

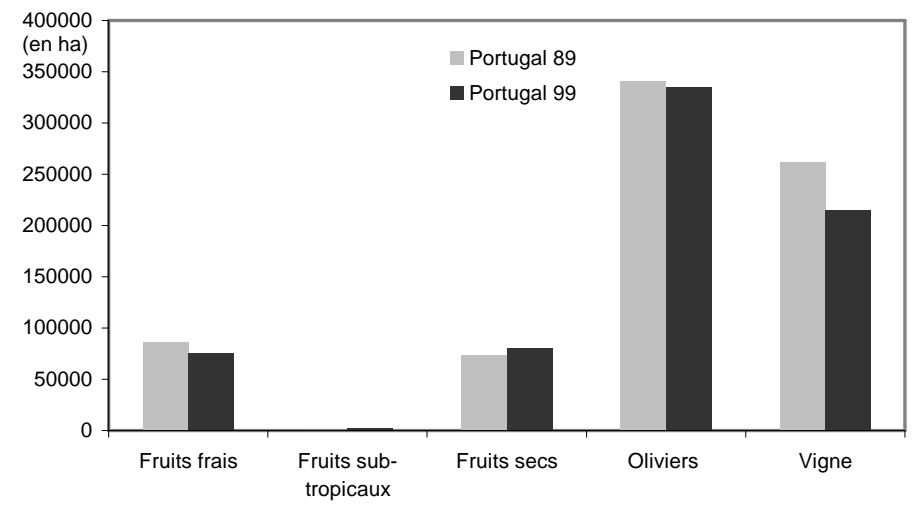

Figure 3. Composition des cultures permanentes au Portugal (ha). (Sources : RGA 1989 et 1999).

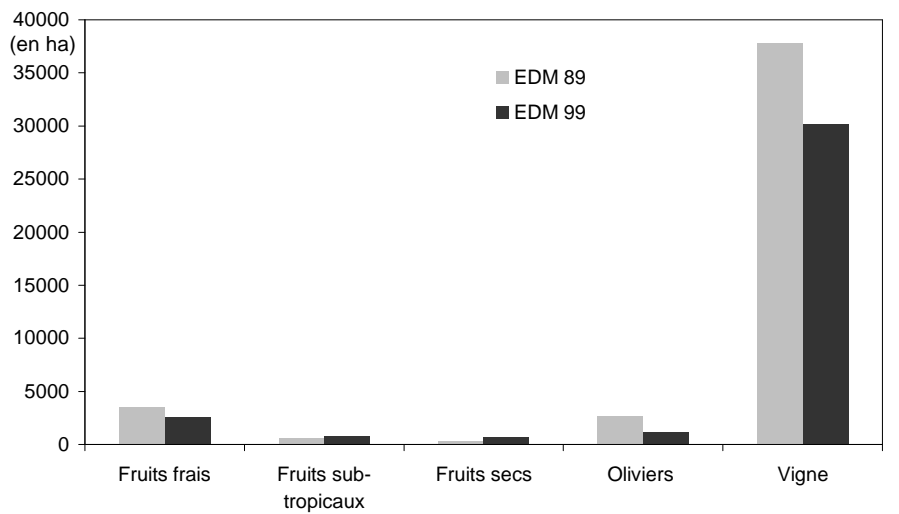

Figure 4. Composition des cultures permanentes dans la région Do Entre Douro e Minho (ha). (Sources : RGA 1989 et 1999). 
Le rapport entre la SAU et la surface totale des fréguesias montre que la surface agricole a diminué sur l'ensemble de la région. Les fréguesias enregistrent une diminution moyenne de $25 \%$ de la SAU (valeur proche de la tendance générale au Portugal). Toutefois, quelques fréguesias enregistrent une augmentation de la SAU entre 1989 et 1999. Il s'agit principalement des secteurs en altitude où les prairies et les pâturages permanents ont subi une augmentation au détriment des secteurs en plaine et en vallée. Cette tendance s'observe principalement dans les fréguesias situées au Nord Est de la région do Entre Douro e Minho dans le parc naturel Peneda Gerês. La relative augmentation de la SAU dans ce secteur montagnard, provient d'une part d'une politique visant à maintenir la production bovine et d'autre part, de l'impossibilité de développer des activités industrielles à cause du statut de parc naturel (fig 5).

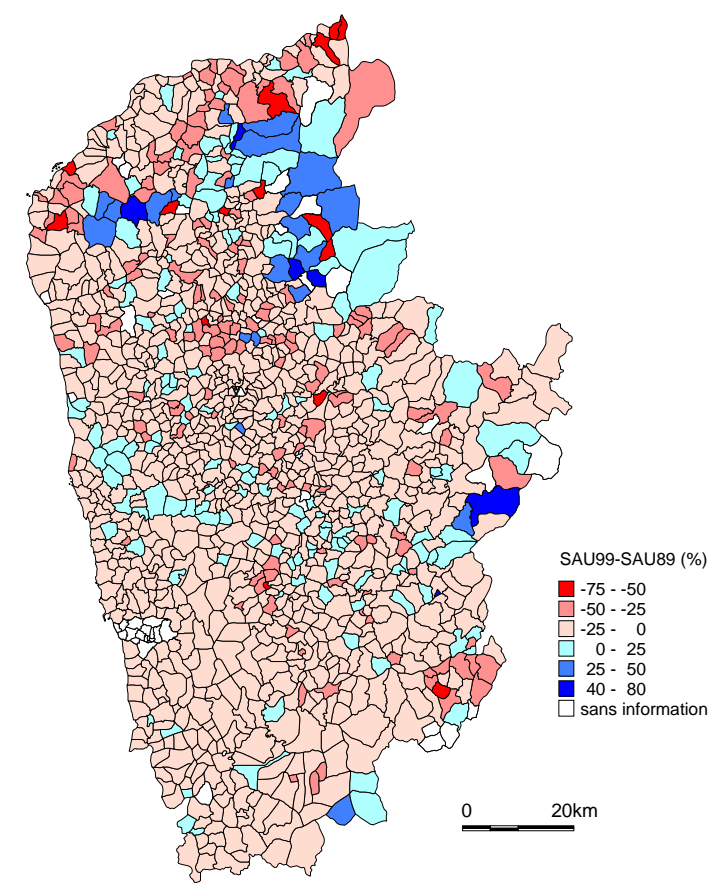

Figure 5. Evolution de la SAU entre 1989 et 1999. (Sources : RGA 1989 et 1999). 
La distribution spatiale du nombre d'exploitations montre une diminution significative et quasi générale sur l'ensemble des fréguesias (seulement 32 fréguesias enregistrent une augmentation entre 1989 et 1999). La baisse du nombre d'exploitations est la plus importante sur le littoral avec la forte expansion urbaine et touristique (Mendes, 1991) (fig 6).

Malgré cette tendance à la baisse du nombre d'exploitations, la dimension moyenne des exploitations augmente (moyenne de 2.6 ha en 1989 et de 3.2 ha en 1999). Nous pouvons en déduire que la variation négative du nombre d'exploitations reflète la diminution du nombre des petites exploitations, qui dans la région, ont une grande importance (Garido, 1989). La polyculture représente encore l'activité agricole principale (culture vivrière), mais elle subit une baisse importante avec l'évolution de l'agriculture de ces dernières années. Depuis le début des années 90 , les politiques agricoles nationales et européennes ont provoquées une restructuration des pratiques agricoles et ont favorisé l'ouverture vers l'exportation dans des domaines tels que l'horticulture ou la viticulture (CEE, 1988 et 1991). Cela se manifeste par la diminution des petites exploitations et du nombre d'agriculteurs dans la région do Entre Douro e Minho.

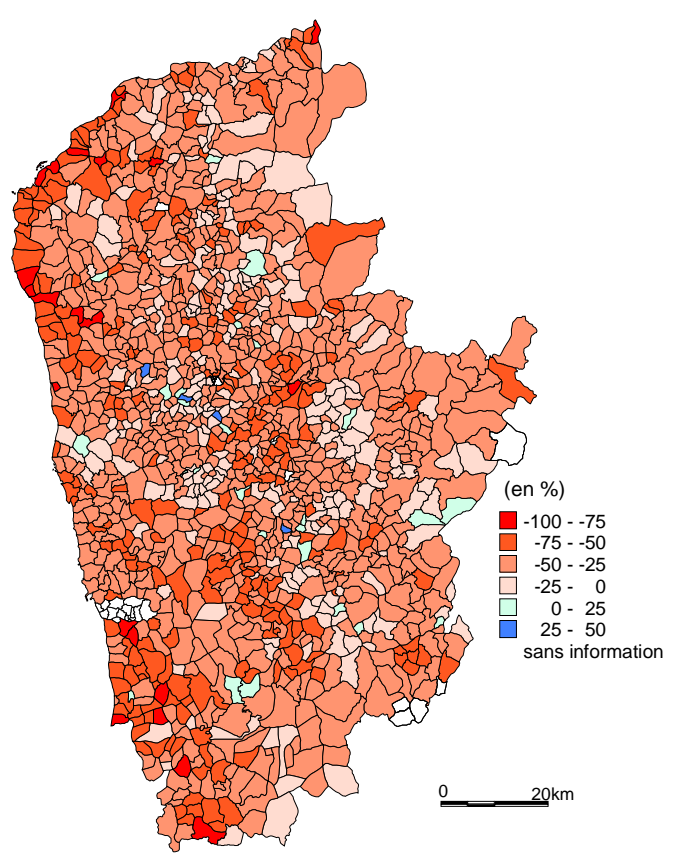

Figure 6. Evolution du nombre d'exploitations agricoles entre 1989 et 1999. 


\subsection{Une diminution du nombre de viticulteurs et une augmentation de la surface en vigne}

En 1999, dans la région DEM, la vigne représente $85 \%$ du total des cultures permanentes et $14 \%$ de la SAU. En 1989, la vigne occupait 37809 hectares avec 100188 exploitations (fig 4). Plus de 93\% des exploitations avaient une superficie inférieure à 1 ha et seulement 34 exploitations avaient une surface égale ou supérieure à 20 ha. La surface en vigne et le nombre d'exploitations viticoles a fortement baissé entre 1989 et 1999. En 1999, les vignobles ne représentent plus qu'une surface de 30231 ha associée à 60273 exploitations, soit une baisse respective de $20 \%$ et $40 \%$. Malgré l'importante diminution de la surface totale cultivée en vigne et du nombre d'exploitations, la surface moyenne des exploitations a augmenté. En 1999, 79\% des exploitations ont une surface en vigne inférieure à 1 ha (-14\% par rapport en 1989). Cette légère augmentation de la surface moyenne des exploitations et la forte baisse du nombre d'agriculteurs mettent en évidence une diminution des petits producteurs pratiquant une agriculture vivrière (polyculture) au profit d'agriculteurs cultivant spécifiquement la vigne dans une optique de grande production.

Au total, la surface de vigne a diminué sur l'ensemble de la région entre 1989 et 1999. Mais, cette tendance n'est pas vérifiée pour l'ensemble des fréguesias. Quelques fréguesias enregistrent une augmentation non négligeable de la surface en vigne. L'évolution de la surface en vigne entre 1989 et 1999 montre que la majorité des fréguesias subit une diminution moyenne entre 0 et 25 ha avec une prédominance au niveau du littoral (ouest) et dans les montagnes (est) (fig 7). Les fréguesias où la surface en vigne a augmenté ne sont pas isolées mais forment des zones spécifiques où le Vinho Verde est cultivé sous la forme de grandes parcelles (au détriment de la culture sous forme de haies). Les deux cartes représentant la surface en vigne par rapport à la SAU en 1989 et 1999, montrent que le «contour géographique » des grandes zones viticoles est similaires pour les deux dates. Pour la majorité de ces fréguesias, la superficie en vigne/SAU a augmenté entre 1989 et 1999. Par exemple, les fréguesias comprenant les communes de Monção et de Melgaço au sud du fleuve Minho (frontière Espagne/Portugal) ont une progression de $25 \%$ de la surface en vigne. Ce secteur produit la variété Alvarinho, vin blanc sucré considéré comme le meilleur Vinho Verde et très apprécié par les connaisseurs (Galhano, 1986 ; Marques, 1987 ; Garrido et Mota, 2001). Dans la vallée du fleuve Tâmega (cf. fig 1) au niveau des communes de Baião et Borne de Canaveses, l'augmentation de la surface en vigne/SAU est supérieure à $50 \%$ (fig 8). Dans ce secteur, c'est également un cépage blanc, l'Avesso, qui connaît un important essor, notamment pour l'exportation (Reis, 2002).

L'utilisation d'indices de statistique spatiale confirme la formation de ces structures spatiales liées à la consolidation du domaine viticole. L'indice de 
ségrégation de Duncan $^{5}$ (0,5 en 1989 et 0,6 en 1999) exprime une distribution hétérogène de la surface en vigne par rapport à la SAU pour l'ensemble des fréguesias. Le quotient de localisation ${ }^{6}$, calculé pour les deux années, met en évidence une concentration supérieure à la moyenne $(>1)$ pour les fréguesias situées dans les vallées et au centre de la région DEM et une concentration inférieure à la moyenne (< 1) sur le littoral et à l'est. Enfin, l'indice du I de Moran ${ }^{7}$ (0,69 en 1989 et 0,65 en 1999) montre la signification statistique des structures spatiales observées aux deux dates.

La figure 9, combinant l'augmentation de la surface en vigne par fréguésia entre 1989 et 1999 et la morphométrie, montre que ce sont les vallées des principaux fleuves (Rio Minho, Rio Lima, Rio Tâmega et Rio Douro) qui ont enregistré la plus forte progression de la surface en vigne entre 1989 et 1999 et plus particulièrement dans la partie intérieure de la région. En effet, la vigne répond à des caractéristiques topoclimatiques spécifiques et les fréguesias soumises aux influences maritimes ne présentent pas les conditions optimales pour obtenir un vin de qualité (Arantes, 1989). De même, les secteurs montagnards ne sont pas favorables car les variations thermiques diurnes et saisonnières sont trop importantes (Daveau, 1988). Par exemple, l'ingénieur agronome Garrido (1984) a démontré que la variété Alvarinho ne pouvait pas être cultivée au-dessus de $200 \mathrm{~m}$ d'altitude. C'est dans la partie est de ces vallées que les vins blancs de Vinho Verde, très prisés et destinés à l'exportation, sont produits (Monteiro et al, 2003). Pour ces fréguesias, la surface en vigne a augmenté entre 10 et 50 ha et même, dans certains cas, de plus de 50 ha (fig 9).

En croisant ces informations avec la carte de l'évolution du nombre d'exploitations (fig 6), nous constatons que les fréguesias qui ont enregistré des augmentations de surface cultivée en vigne ont connu, dans leur majorité, une forte diminution du nombre d'exploitations. Cela démontre d'une part, que la viticulture devient l'activité principale du secteur agricole de ces fréguesias et d'autre part que la surface cultivée de ces exploitations augmente significativement. On note donc la disparition des petites exploitations à vocation vivrière qui cultivaient un Vinho Verde destiné à la production locale. Avec l'amélioration des techniques de culture, de la qualité du Vinho Verde et l'orientation économique vers l'exportation, les grandes exploitations se sont développées dans les secteurs topoclimatiques favorables (coteaux de vallées protégés de l'influence océanique), c'est-à-dire dans la partie Est des vallées des grands fleuves. Cette évolution a fortement modifié les pratiques culturales et les terroirs de la région do Entre Douro e Minho.

\footnotetext{
${ }^{5}$ L'indice de ségrégation de Duncan mesure la distribution d'un groupe et varie de 0 à 1 , valeurs qui correspondent respectivement à une distribution parfaitement égale dans les unités spatiales et à une ségrégation maximale (Jakubs, 1981).

${ }^{6}$ Le quotient de localisation est le rapport entre la proportion du groupe dans l'unité spatiale et la proportion du groupe dans la région. Si le quotient est $>1$, le groupe est sur-représenté dans l'unité spatiale et inversement s'il est $<1$ (Apparicio, 2000).

${ }^{7}$ L'indice de Moran vise à mesurer si deux unités "proches" se ressemblent plus que deux unités quelconques (0 signifie "éloigné" et 1 "proche") (Moran, 1948).
} 


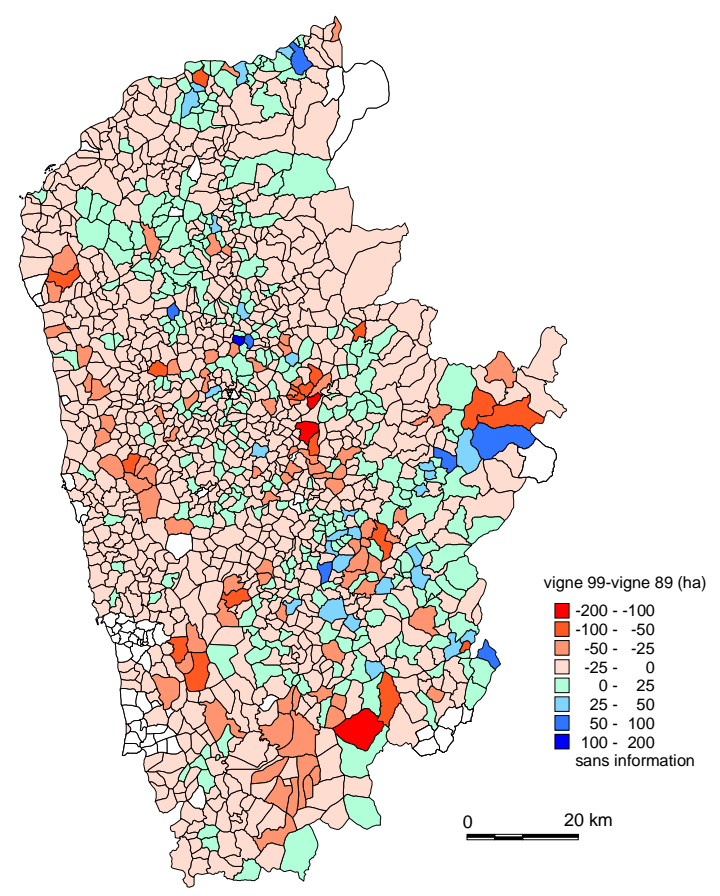

Figure 7. Evolution de la surface en vigne entre 1989 et 1999.
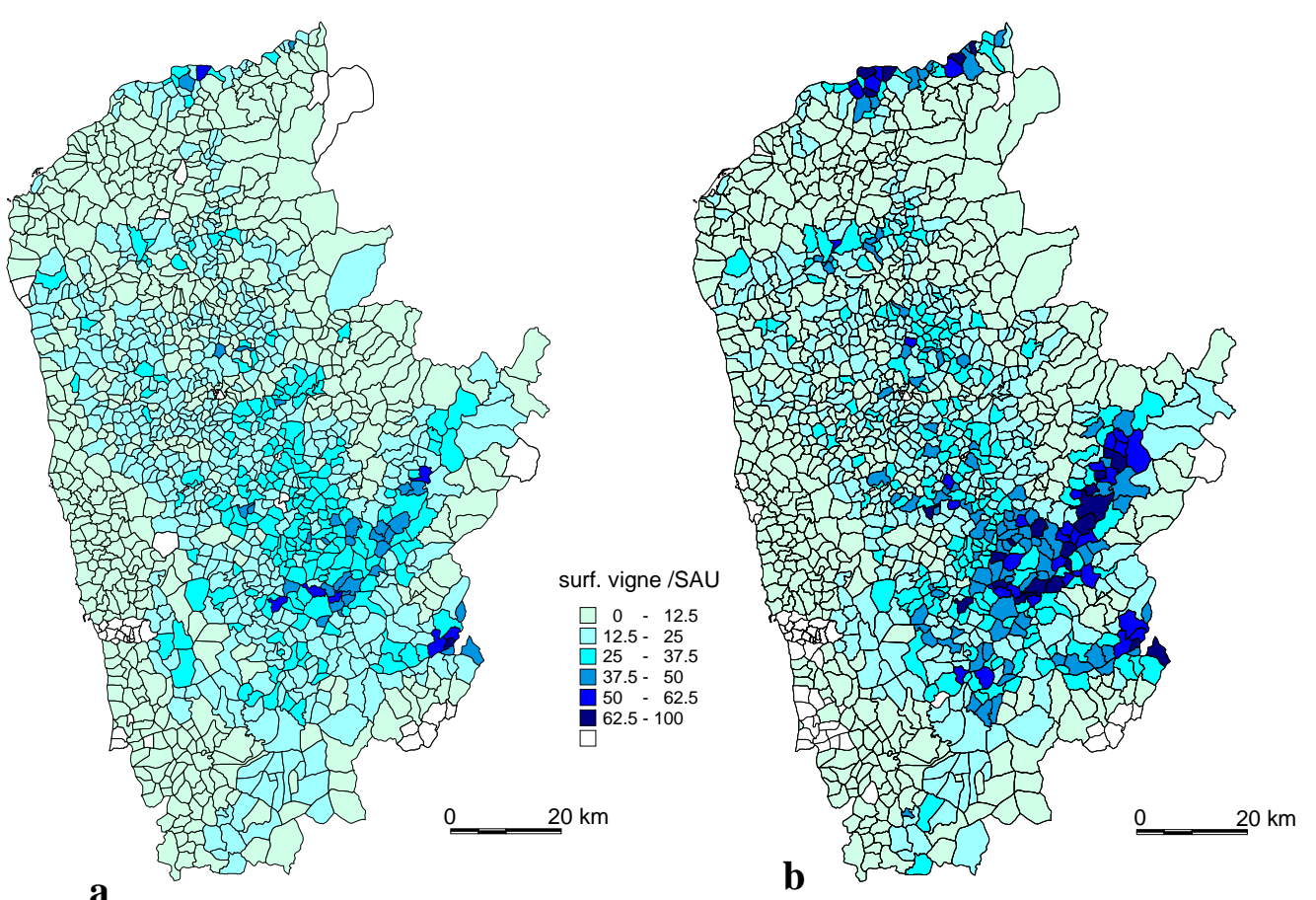

Figure 8. Surface en vigne (en ha) par rapport à la SAU en 1989 (a) et en 1999 (b). 

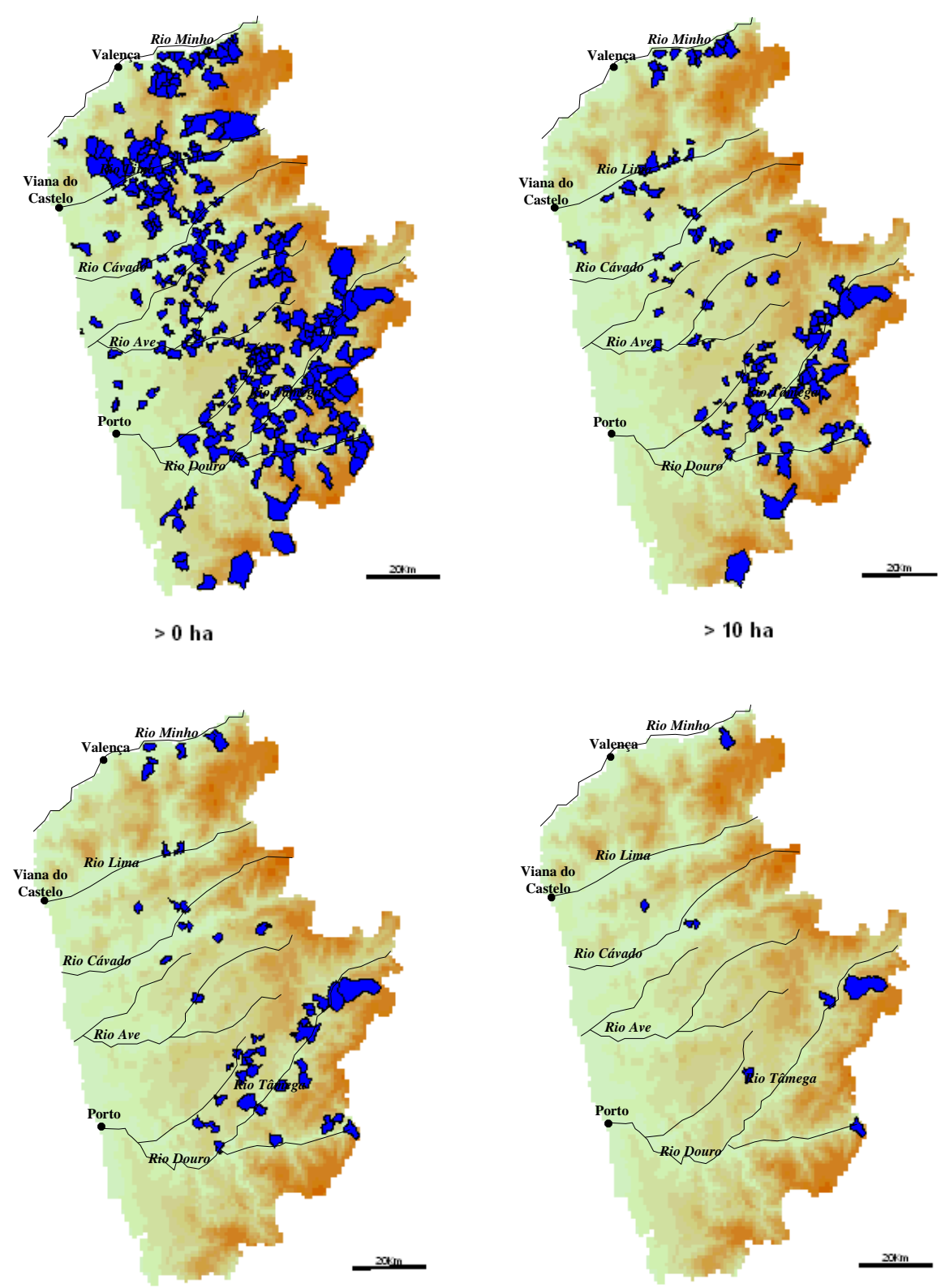

$>20$ ha

$>50$ ha

En bleu : fréguesias où l'on enregistrent une augmentation de la surface en vigne

Figure 6. Augmentation de la surface en vigne entre 1989 et 1999. 


\section{Evolution des pratiques culturales et transformation des territoires ruraux}

L'analyse des données des RGA de 1989 et 1999 a montré une baisse importante de la part relative de l'agriculture (SAU et nombre d'agriculteurs) dans la région do Entre Douro e Minho. Celle-ci, due à l'essor de l'industrialisation et aux diverses politiques agricoles, a complètement modifié le paysage agricole. L'évolution de la culture du Vinho Verde est le parfait exemple pour évoquer la transformation des pratiques agricoles et des territoires ruraux. Entre 1989 et 1999, nous avons remarqué une diminution générale de la surface cultivée en vigne mais celle-ci est très hétérogène selon les secteurs : augmentation significative de la surface en vigne dans la partie intérieure des vallées alors que le nombre d'exploitations de ces fréguesias a fortement diminué.

Il y a quelques années, le Vinho Verde était encore cultivé de manière traditionnelle. "Reléguée aux limites des champs cultivés ou dans les zones mortes des exploitations", la vigne était plantée sous forme de petites treilles traditionnelles appelées "enforcado" ou "arejão" (Galhano, 1986 et 1989). Ce mode de conduite permettait aux agriculteurs de produire suffisamment de vin pour la consommation familiale en utilisant un minimum de surface dans l'optique d'une agriculture vivrière. Mais l'évolution de la conjoncture économique (crise de l'agriculture dans les années 1980) et les différentes politiques agricoles (européennes et nationales) ont engendré le départ des "petits" agriculteurs vers l'industrie et l'avènement d'une viticulture plus moderne, plus productive pratiquée par une minorité de viticulteurs. Le passage de la culture vivrière vers la production spécifique d'un Vinho Verde de grande qualité a nécessité la création de structures : groupement en coopératives, création de la Comissão de Viticultura da Região dos Vinhos Verdes et création d'une station d'expérimentation scientifique du Vinho Verde financée par l'ensemble des producteurs. Cela a permis d'augmenter considérablement la quantité de la production, d'améliorer la qualité du Vinho Verde et de mettre en valeur l'appellation d'origine contrôlée "Vinho Verde". Dans une politique exportatrice, les modes de conduite de la vigne ont évolué - passage de la treille à la parcelle de vigne - et ont donc complètement modifié les territoires ruraux. Par conséquent, d'une viticulture dispersée, avec des vignes en bordure de champs (photo 1), essentiellement tournée vers la production de vins rouges ${ }^{8}$ très appréciés par la population locale, la culture moderne du Vinho Verde s'est orientée vers des terroirs continus avec des modes de conduite (palissage en Cruzeta) privilégiant la mécanisation et produisant des vins blancs dont les caractéristiques correspondent plus à la demande extérieure.

L'amélioration de la qualité du Vinho Verde et la forte demande a provoqué l'extension des vignobles. Les terroirs se sont développés à flanc de coteau utilisant

8. Traditionnellement, les Vinho Verde sont des vins rouges pouvant aller jusqu'au grenat moyennement alcoolisés avec une forte acidité. Les Vinho Verde blancs, plus récents, plus fruités avec des taux d'alcool plus élevées et moins d'acidité s'exportent beaucoup mieux. 
ainsi des secteurs forestiers. Les viticulteurs ont fait de la production de Vinho Verde leur activité principale. Les grands châteaux (ex: Palácio da Breloeira) producteurs de Vinho Verde depuis plusieurs décennies se sont modernisés, produisant, vinifiant et commercialisant ainsi des vins de luxe très renommés. Les "nouveaux" viticulteurs ont développés leurs exploitations en s'inspirant des techniques des pays grands producteurs de vin. Soit ils produisent le raisin, le vinifie et commercialisent directement soit ils le livrent dans des coopératives qui vinifient et commercialisent le vin suivant les structures établies.

La politique agricole européenne a grandement influencée la transformation des territoires ruraux da la région do Entre Douro e Minho. D'ailleurs en 1986, Galhano indiquait, "actuellement, l'agriculture de l'Entre Douro e Minho compose un paysage aimable et verdoyant: champs céréaliers où domine presque exclusivement le maïs et le "ray-grass", vignes bordant les champs où les cultures se succèdent sans interruption. Ce paysage a du être profondément modifié par l'introduction du maïs en Europe. Il est difficile d'imaginer ce qu'était l'agriculture du Minho - et d'ailleurs - avant l'expansion de cette plante. Seule la vigne demeure en sa forme et sa localisation primitives, aujourd'hui socialement et économiquement dépassées et qui seront, peu à peu, remplacées par des méthodes et des localisations répondant mieux aux conditions actuelles...".

En 1988, l'application du Règlement 2729/88 de la CEE concernant l'attribution de primes pour l'abandon de la vigne, a provoqué une forte diminution de la surface de vigne (notamment dans les fréguesias près du littoral) et par conséquent une baisse importante du nombre d'agriculteurs qui se sont principalement reconvertis dans le domaine industriel. En 1991, les politiques successives de la PAC ont apporté des aides pour améliorer et restructurer le secteur viticole au Portugal. Les conséquences pour la région EDM ont été immédiates avec le développement de la production moderne du Vinho Verde dans les secteurs où les conditions géographiques et climatiques étaient favorables à production d'un Vinho Verde de qualité (partie intérieure des vallées des grands fleuves). Profitant du statut prestigieux du Vinho Verde au Portugal (grands châteaux connus depuis plusieurs siècles ; appellation Vinho Verde depuis 1908), la région EDM a profité de ces aides pour développer l'image du Vinho Verde (Mendes, 1991). Par exemple, à travers le Règlement 2323/91 qui soutenait l'investissement dans les exploitations agricoles, la viticulture régionale a investi, dans la période comprise entre 1986 et 1993, principalement dans les communes localisées entre les fleuves Minho et Lima (Pont de Lime, Monção et de Melgaço), et dans les communes au niveau des fleuves Douro et Tâmega (Amarante, Baião, Cinfães, M. Canaveses). La réorganisation et la modernisation du secteur Vinho Verde dans ces fréguesias ont été poursuivies avec de nouveaux programmes européens comme le Programa Operacional da Vinha entre 1991 et 1993, le PAMAF (période 1994-1999) ou le VITIS visant à améliorer les structures viticoles et l'exportation.

Actuellement, le seul frein au développement du Vinho Verde dans ces secteurs, est la difficulté naissante dans la commercialisation du vin: diminution de la 
consommation, persistence de productions de basse qualité notamment ces dernières années qui ont été des années de grande production (augmentations des stocks) et encore de l'arrivée sur la marché de vins provenant de nouveaux pays à des prix concurrentiels (Amorim, 1991; Dubos, 1991; Thrift, 1997). Les exigences du marché national et mondial peuvent faire rapidement évoluer l'essor actuel du Vinho Verde et par conséquent transformer les territoires ruraux.

\section{Conclusion}

L'intégration des données RGA 1989 et 1999 dans un SIG composé des limites administratives des fréguesias et de la morphométrie a permis de réaliser une représentation spatiale de l'évolution de l'agriculture et plus particulièrement de la viticulture dans la région do Entre Douro e Minho. L'analyse des cartes montre une diminution générale la surface en vigne et du nombre d'exploitations sur l'ensemble de la région. Toutefois, certaines fréguesias enregistrent un accroissement de la surface en vigne avec paradoxalement une forte diminution $\mathrm{du}$ nombre d'exploitations. Cette évolution correspond à l'essor actuel de la production de Vinho Verde dans certains secteurs bien délimités de la région (ex : vallée du Minho, vallée du Lima, ...). Ici, le SIG a permis de visualiser spatialement les grandes zones regroupant les fréguesias où le développement du Vinho Verde s'opère, c'est-à-dire la partie intérieure des grandes vallées.

Cette étude a montré que les outils de la géomatique permettent de cartographier l'évolution des territoires ruraux. Dans ce travail, le SIG a permis d'observer ces transformations à l'échelle des fréguesias. Pour améliorer et affiner les résultats, nous comptons utiliser des images satellitaires multi-date à haute résolution spatiale (10 mètres) de manière à réaliser une cartographie précise de l'évolution des grandes parcelles de Vinho Verde.

\section{Bibliographie}

Amorim, P., Vinho Verde na Exportação. Que Futuro?, A Revista do Vinho Verde, Comissão de Viticultura da Região dos Vinhos Verdes, n. ${ }^{\circ}$ 6, Porto, Abril/Maio/Junho de 1991.

Apparicio, P., Les indices de ségrégations résidentielle : un outil intégré dans un Système d'Information Géographique. Cybergéo, n³4, 2000.

Arantes, J., O Noroeste Vitícola Ontem e Hoje, A Revista do Vinho Verde, Comissão de Viticultura da Região dos Vinhos Verdes, Porto, Maio de 1989.

Bridier, S., Quénol, H. et Beltrando, G., Simulation du potentiel de refroidissement en situation radiative: application aux terroirs des Fonds de Sillery dans le vignoble de Champagne. Revue Internationale de Géomatique, 2004. (sous presse). 
16 Revue internationale de Géomatique.- n X/2005

Castro R., Santos A., Adequaçao da viticultura etrusca a poda mecanica da Emilia Romana aos vinhos verdes, Congresso Ibérico de Ciências Horticolas - Lisboa 18-21 Junho 1990 , p. 327-334.

Daveau S., Geografia de Portugal - O Ritmo Climático e a Paisagem, Edições João Sá da Costa, Lisboa, 1988.

Dubos J., $14^{\circ}$ Relatório de Conjuntura Vitivinícola Mundial - Excedentes em Quantidade Preocupante, A Revista do Vinho Verde, n. ${ }^{\circ}$ 7, Comissão de Viticultura da Região dos Vinhos Verdes, Porto, Julho/Agosto/Setembro, 1991.

Galhano A., Le vinho verde : une région délimitée, une appelation d'origine, Comissão de Viticultura da Região dos Vinhos Verdes, 1986.

Galhano, A., Região do Vinho Verde Face à Integração Europeia (2), A Revista do Vinho Verde, n. ${ }^{\circ}$ 1, Comissão de Viticultura da Região dos Vinhos Verdes, Porto, Agosto de 1989.

Garido J., Zonagem viticola da Sub-Região de Monção, caso do "Alvarinho”, 1984, Univ. Vila Real.

Garrido J. et Mota T, Implantação da vinha, Manuel Técnico, EVAG 2001, 35p.

Instituto National de Estatistica, Données RGA 1989 et 1999, CDRom, 2000.

Jakubs J.P., A distance based segregation index. Journal of Socio-Economic Planning Science, 15, pp 129-141.

Marques H., Região Demarcada dos Vinhos Verdes, Revista da Faculdade de Letras Geografia, I Série, Volume III, Porto, 1987, pp. 135-242.

Mendes, A. M. C., Evolução da Estrutura da Vitivinicultura da Região dos Vinhos Verdes no Período 1953-86 e suas Perspectivas Futuras no Quadro do Regulamento CEE 2239/86, A Revista do Vinho Verde, n. ${ }^{\circ}$ 7, Comissão de Viticultura da Região dos Vinhos Verdes, Porto, Julho/Agosto/Setembro, 1991.

Monteiro A., Ferreira C., Madureira H., Pinto A., Ramadas I., Guerner J., Atlas Agroclimatológico do Entre Douro e Minho - Relatório Preliminar, Porto, 2001, polic.

Monteiro A., Ferreira C., Madureira H., Pinto A., Ramadas I., Guerner J., Atlas Agroclimatológico do Entre Douro e MinhoRelatório Projecto POCTI/GEO/14260/1998, Porto, 2003.

Moran P., The interpretation of statistical maps, Journal of Royal Statistical Society, vol. 10B, 1948, pp 243-251.

Regulamento (CEE) n²729/88 Da Comissão de 31 de Agosto de 1988, Regulamento relativo à concessão para as campanhas vitícolas de 1988/1989 a 1995/1996 de prémios de abandono definitivo de superfícies vitícolas, Journal Oficial das Comunidades Europeias.

Regulamento (CEE) n²328/91 Do Conselho de 15 Julho de 1991, Regulamento relativo à melhoria da eficácia das estruturas agrícolas, Journal Oficial das Comunidades Europeias.

Reis J.L., An Região demarcada dos vinhos verdes : um século de història, Comissão de Viticultura da Região dos Vinhos Verdes, 2002. 
Thrift, N.J., Conceptualising the evolution of the European Union's agri-environment policy: a discourse approach, Environment and planning, volume 29, $\mathrm{n}^{\circ}$ 10, October 1997, p.1869-1885.

VITIS, Regime de Apoio à reconversão e reestruturação das vinhas vitis, Ministério da Agricultura, do Desenvolvimento Rural e das Pescas,1999.

SERVICE EDITORIAL HERMES - LAVOISIER

14 RUE DE PROVIGNY - 94236 CACHAN

Tel : 01-47-40-67-00 Télécopie : 01-47-40-67-02

E-mail : revues@lavoisier.fr

Serveur Web : http//www.hermes-science.com 